\title{
VII. INTERSTITIAL WATER RESULTS, LEG 42A
}

\author{
G. W. Bode and V. S. Sotelo, Deep Sea Drilling Project, Scripps Institution of Oceanography, La Jolla, \\ California
}

\section{INTRODUCTION}

Interstitial water samples were routinely analyzed on board by V. S. Sotelo. Analyses included determinations of $p \mathrm{H}$, salinity, chlorinity, alkalinity, calcium, and magnesium.

\section{SAMPLING}

Interstitial water samples were taken at regular intervals and where major changes in lithology occurred. Sampling was effected by removing a $10-\mathrm{cm}$ minicore from either end of a section. Care was taken not to sample lithologic boundaries, the top of Section 1 or the bottom of Section 6 (top or bottom of core) or their equivalents in cores with less than full recovery.

The mini-core was then capped, labeled, and refrigerated until the analyst could process it.

\section{SAMPLE PREPARATION}

All mini-cores were allowed to reach room temperature (required about 3 hours) before they were processed. When the temperature had reached equilibrium, the sediment was extruded onto a stainless steel tray and the outer deformed edges of the core carefully cut away. The cleaned sediment was then placed in a Manheim squeezer and squeezed with up to 24,000 pounds pressure in the more indurated sediments. The released water was collected in a syringe and then filtered using a millipore filter.

\section{ANALYTICAL PROCEDURES}

The procedures used for shipboard analysis have undergone little change since they were described by Gieskes (1973) and Gieskes and Rogers (1973). The main differences is that all work is now done at room temperature rather than at thermostatically controlled temperatures and some sample sizes are smaller to conserve material. Also a non acid washed filter paper is used to prevent the titration of the sample during the squeezing process.

All interstitial water not used was sealed in polyethylene tubes and/or glass ampules and sent to DSDP for storage.

\section{RESULTS}

The results are listed in Table 1 and are displayed graphically in the individual site reports.

\section{REFERENCES}

Gieskes, J. M., 1973. Interstitial water studies, Leg 15 , alkalinity, $p \mathrm{H}, \mathrm{Mg}, \mathrm{Ca}, \mathrm{Si}, \mathrm{PO}_{4}$, and $\mathrm{NH}_{4}$. In Heezen, $\mathrm{B}$. C., MacGregor, I. G., et al., Initial Reports of the Deep Sea Drilling Project, Volume 20: Washington (U.S. Government Printing Office), p. 813-830.

Gieskes, J. M., and Rogers, W. C., 1973. Alkalinity determination of interstitial water of marine sediments. J. Sediment. Petrol., v. 43, p. 272-277. 
TABLE 1

Results of the Interstitial Water Determinations, Leg 42A

\begin{tabular}{|c|c|c|c|c|c|c|c|}
\hline $\begin{array}{l}\text { Sample (Interval } \\
\text { in } \mathrm{cm} \text { ) }\end{array}$ & $\begin{array}{l}\text { Depth } \\
\text { (m) }\end{array}$ & $p \mathrm{H}$ & $\begin{array}{l}\text { Alkalinity } \\
\text { (meq/1) }\end{array}$ & $\begin{array}{l}\text { Salinity } \\
(\% \circ)\end{array}$ & $\underset{(\mathrm{mmoles} / \mathrm{l})}{\mathrm{Ca}^{++}}$ & $\underset{(\mathrm{mmoles} / \mathrm{l})}{\mathrm{Mg}^{++}}$ & $\begin{array}{l}\mathrm{CL}^{-} \\
(\% \circ)\end{array}$ \\
\hline \multicolumn{8}{|l|}{ Site 371} \\
\hline IAPSO Seawater & - & 7.78 & 2.30 & 35.2 & 10.6 & 54.0 & 19.4 \\
\hline Surface Seawater & - & 8.05 & 2.37 & 37.4 & 11.2 & 58.2 & 20.4 \\
\hline $1-5,144-150$ & 8.0 & 6.90 & 3.58 & 40.7 & 14.2 & 63.6 & 22.3 \\
\hline $2-2,144-150$ & 207.44 & 5.99 & 0.633 & 103.0 & 219.8 & 188.3 & 57.8 \\
\hline $3-5,144-150$ & 368.94 & 8.16 & 0.567 & 133.6 & 335.1 & 264.6 & 76.2 \\
\hline $4-5,146-150$ & 406.96 & 6.18 & 0.554 & 185.9 & 536.3 & 440.2 & 100.5 \\
\hline $5-5,140-150$ & 463.9 & 5.76 & 0.569 & 253.0 & 691.6 & 633.9 & 127.2 \\
\hline $6, \mathrm{CC}$ & 518.0 & - & - & 286.0 & 867.4 & 944.4 & 150.2 \\
\hline $8-2,140-150$ & 549.4 & 5.27 & 2.91 & 332.2 & 1380.0 & 894.8 & 170.4 \\
\hline
\end{tabular}

Site 372

$\begin{array}{lcclcccc}\text { IAPSO Seawater } & - & 7.78 & 2.30 & 35.2 & 10.6 & 53.9 & 19.3 \\ \text { Surface Seawater } & - & 7.99 & 2.39 & 37.4 & 11.0 & 57.76 & 20.1 \\ 1-3,144-150 & 119.94 & 6.82 & 2.37 & 55.0 & 35.6 & 77.3 & 29.9 \\ 2-3,144-150 & 138.94 & 6.85 & 1.61 & 58.3 & 47.9 & 73.7 & 31.6 \\ 3-2,144-150 & 148.44 & 6.92 & 1.36 & 59.4 & 52.8 & 74.6 & 31.9 \\ 9-3,140-150 & 203.40 & 6.83 & 1.131 & 74.8 & 84.4 & 83.5 & 42.4 \\ 14-4,140-150 & 251.40 & 7.07 & 0.752 & 73.4 & 71.9 & 69.3 & 42.5 \\ 19-4,140-150 & 298.90 & 7.46 & 0.576 & 78.1 & 79.5 & 57.1 & 44.0 \\ 24-5,140-150 & 347.90 & 6.94 & 0.762 & 83.1 & 79.7 & 59.2 & 48.4 \\ 29-4,140-150 & 395.40 & 7.15 & 0.743 & 90.2 & 100.2 & 61.8 & 58.5 \\ 33-5,140-150 & 471.40 & 6.98 & 0.860 & 95.7 & 104.1 & 65.4 & 57.1 \\ 35-2,140-150 & 536.90 & - & - & 104.5 & 116.4 & 69.9 & 62.7 \\ 36-5,140-150 & 579.0 & 7.06 & 0.879 & 106.7 & 118.0 & 65.4 & 63.7 \\ 38-3,140-150 & 648.9 & - & - & 114.4 & 131.1 & 72.1 & 69.3 \\ 40-4,140-150 & 727.9 & - & - & & \text { No Pore Water Obtained } & \\ 42-5,140-150 & 794.4 & - & - & 117.7 & 128.9 & 63.8 & 71.1 \\ 43-5,140-150 & 806.9 & - & - & 123.2 & 135.8 & 77.2 & 74.5 \\ 44-5,40-50 & 840.9 & - & - & 112.2 & 120.4 & 85.1 & 68.1 \\ 45-3,140-150 & 876.9 & - & - & 110.0 & 118.8 & 72.2 & 66.5\end{array}$

Site 374

$\begin{array}{lrllrrrr}\text { IAPSO Seawater } & - & 7.72 & 2.35 & 35.2 & 10.6 & 54.0 & 19.4 \\ \text { Surface Seawater } & - & 8.08 & 2.57 & 38.5 & 11.2 & 60.4 & 21.3 \\ 2-2,140-150 & 159.9 & 7.56 & 3.70 & 57.7 & 17.7 & 93.0 & 34.0 \\ 4-3,140-150 & 254.9 & 5.5 & 0.889 & 139.7 & 133.5 & 528.0 & 78.0 \\ 5-4,140-150 & 302.4 & 4.9 & 0.406 & 247.5 & 267.0 & 1163.0 & 133.0 \\ 6-1,140-150 & 332.4 & 4.8 & 0.337 & 242.0 & 359.2 & 1028.1 & 126.0 \\ 6-5,140-150 & 338.4 & 5.1 & 0.352 & 240.0 & 372.0 & 1052.0 & 125.0 \\ 7-1,140-150 & 341.9 & 6.0 & 1.56 & 336.6 & 489.7 & 1329.0 & 151.0 \\ 7-2,140-150 & 343.4 & - & - & 290.4 & 508.4 & 1355.0 & 154.0 \\ 7-3,140-150 & 344.9 & - & - & 341.0 & 495.4 & 1371.0 & 150.0 \\ 7-4,140-150 & 346.4 & 5.6 & 1.72 & 288.0 & 503.5 & 1363.0 & 154.0 \\ 7-5,140-150 & 347.8 & 5.4 & 1.68 & 367.4 & 523.1 & 1420.0 & 161.0 \\ 7-6,140-150 & 349.5 & 6.0 & 1.62 & 362.0 & 493.8 & 1347.0 & 151.0 \\ 8-2,140-150 & 355.9 & 6.0 & 2.03 & 352.0 & 452.4 & 1309.0 & 144.0 \\ 9-3,140-150 & 366.9 & 5.9 & 2.97 & 371.8 & 477.0 & 1598.0 & 162.0 \\ 13-1,140-150 & 389.4 & 5.6 & 3.42 & 387.2 & 025.5 & 2162.0 & 165.0 \\ 13-3,0-10 & 391.0 & - & - & 290.0 & 10.8 & 1428.0 & 112.0 \\ 14-1,140-150 & 395.4 & 5.3 & 4.86 & 407.0 & 10.3 & 2268.0 & 171.0 \\ 15-1,140-150 & 404.9 & 5.3 & 4.90 & 462.0 & 10.3 & 2271.0 & 173.0\end{array}$

Site $\mathbf{3 7 5}$

\begin{tabular}{lccccccr} 
Surface Seawater & - & 8.09 & 2.57 & 38.5 & 11.4 & 58.8 & 21.4 \\
$5-3,140-150$ & 364.9 & 7.95 & 3.66 & 33.0 & 12.3 & 25.3 & 20.4 \\
$6-5,140-150$ & 468.4 & - & 3.23 & 31.9 & 13.0 & 18.2 & 19.4 \\
$7-5,140-150$ & 572.9 & 8.097 & 3.7 & 31.6 & 12.6 & 17.9 & 19.0 \\
$8-5,140-150$ & 630.9 & - & - & 28.6 & 14.2 & 17.3 & 17.1 \\
& & & & & & & \\
Site 376 & & & & & & & \\
& & & & & & & \\
IAPSO Seawater & - & 7.7 & 2.34 & 35.2 & 10.6 & 53.9 & 19.4 \\
Surface Seawater & - & 8.0 & 2.58 & 39.0 & 11.8 & 94.4 & 21.4 \\
\hline
\end{tabular}


TABLE 1 - Continued

\begin{tabular}{|c|c|c|c|c|c|c|c|}
\hline $\begin{array}{l}\text { Sample (Interval } \\
\text { in } \mathrm{cm} \text { ) }\end{array}$ & $\begin{array}{l}\text { Depth } \\
\text { (m) }\end{array}$ & $p \mathrm{H}$ & $\begin{array}{l}\text { Alkalinity } \\
(\mathrm{meq} / \mathrm{l})\end{array}$ & $\begin{array}{c}\text { Salinity } \\
(\% \circ)\end{array}$ & $\underset{(\mathrm{mmoles} / \mathrm{l})}{\mathrm{Ca}^{++}}$ & $\underset{\text { (mmoles/l) }}{\mathrm{Mg}^{++}}$ & $\begin{array}{l}\mathrm{CL}^{-} \\
(\% \circ)\end{array}$ \\
\hline $1-4,144-150$ & 5.94 & 8.0 & None & 38.5 & 10.5 & 57.4 & 21.2 \\
\hline $5-4,144-150$ & 43.94 & 7.7 & 3.02 & 38.0 & 11.3 & 55.0 & 21.1 \\
\hline $9-3,144-150$ & 81.94 & 7.6 & 1.44 & 38.5 & 16.3 & 55.3 & 20.9 \\
\hline $12-3,140-150$ & 108.90 & 7.6 & 1.27 & 38.5 & 24.3 & 52.5 & 20.7 \\
\hline $13-2,140-150$ & 118.40 & 7.7 & 1.33 & 38.5 & 27.7 & 53.2 & 20.8 \\
\hline $15-2,140-150$ & 129.40 & 7.8 & 0.61 & 42.4 & 38.3 & 55.8 & 21.8 \\
\hline \multicolumn{8}{|l|}{ Site 377} \\
\hline IAPSO Seawater & - & 7.6 & 2.30 & 35.2 & 10.5 & 53.9 & 19.4 \\
\hline Surface Seawater & - & 8.1 & 2.60 & 38.5 & 11.5 & 60.0 & 21.8 \\
\hline $1-1,140-150$ & 19.19 & 7.4 & 5.20 & 37.4 & 07.1 & 38.3 & 22.6 \\
\hline \multicolumn{8}{|l|}{ Hole 378} \\
\hline IAPSO Seawater & - & 7.6 & 2.30 & 35.2 & 10.6 & 53.9 & 19.4 \\
\hline Surface Seawater & - & 8.1 & 2.58 & 39.0 & 11.4 & 60.7 & 22.4 \\
\hline $1-2,0-6$ & 92.0 & 7.5 & 3.32 & 35.2 & 10.9 & 38.0 & 21.0 \\
\hline $5-1,140-150$ & 129.9 & 7.3 & 3.40 & 36.3 & 13.3 & 40.9 & 21.3 \\
\hline $7-4,140-150$ & 177.4 & 7.5 & 1.87 & 35.8 & 15.1 & 36.1 & 20.9 \\
\hline $8-1,0-10$ & 223.5 & 7.2 & 2.62 & 35.8 & 15.3 & 37.2 & 20.9 \\
\hline $11-3,140-150$ & 310.4 & 7.6 & 0.62 & 41.2 & 40.5 & 44.7 & 20.9 \\
\hline \multicolumn{8}{|l|}{ Hole 378A } \\
\hline $1-2,140-150$ & 50.9 & 7.5 & 4.13 & 36.3 & 07.4 & 44.9 & 21.3 \\
\hline $3-5,140-150$ & 300.4 & 7.7 & 0.630 & 40.7 & 40.5 & 43.7 & 21.3 \\
\hline
\end{tabular}

